\title{
Task Assignment and Pricing Based on Psychological Surplus Value Model
}

\author{
Qiao-Zhen $\mathrm{Yu}^{1,2}$, Zi-Ang Lin ${ }^{1,3}$, Shao-Zhen Chen ${ }^{1,4}, \&$ Yuan-Biao Zhang ${ }^{1,5}$ \\ ${ }^{1}$ Innovation Practice Base of Mathematical Modeling of Jinan University, Zhuhai, China \\ ${ }^{2}$ Software Engineering, Electrical and Information College of Jinan University, Zhuhai, China \\ ${ }^{3}$ Financial Management, International Business College of Jinan University, Zhuhai, China \\ ${ }^{4}$ Finance Department, International Business College of Jinan University, Zhuhai, China \\ ${ }^{5}$ Key Laboratory of Product Packaging and Logistics of Guangdong Higher Education Institutes, Jinan \\ University, Zhuhai, China \\ Correspondence: Yuan-Biao Zhang, Innovation Practice Base of Mathematical Modeling, Electrical and \\ Information College of Jinan University, Zhuhai 519070, China. E-mail: zybt@jnu.edu.cn
}

Received: October 24, 2017

doi:10.5539/ijbm.v13n6p267
Accepted: May 3, 2018

Online Published: May 15, 2018

\begin{abstract}
"Take photos to make money" APP is a self-service crowdsourcing platform relying on mobile Internet, which provides various business inspection and information collection for enterprises. An excellent crowdsourcing platform needs to meet a lot of conditions, and whether the allocation of tasks and pricing is reasonable is the key. In this paper, we set up the task allocation and pricing model based on UTM projection principle, urban residents travel speed weighting method, k-means clustering, ant colony algorithm and comprehensive distance value theory. Then we use the data of "China Undergraduate Mathematical Contest in Modeling" in 2017 to test the model, which proves the robustness of the model in practical application.
\end{abstract}

Keywords: task pricing, psychological residual value, k-means clustering, Hamiltonian circuit, ant colony algorithm

\section{Introduction}

Now, "take pictures to make money" has become a mobile Internet under a self-service model. Compared with the traditional market survey, this mobile Internet-based self-service package can provide enterprises with more convenient and effective business inspection and information collection. However, in the actual crowdsourcing platform, but there is a task allocation inefficient and unreasonable pricing situation, which resulted in the task after the release of nobody cares about the situation.

The core of the package is to establish a set of task allocation mechanism and its matching pricing method, the current research in this area has the following results: According to the method of assigning the task of crowdsourcing tasks, Jun $\mathrm{Wu}$ (2015) classifies the task of the package from the two dimensions of innovation degree and technical content, and then selects the task duration, the turnover amount, the number of auctions, the repeatability and the qualification Feature observation indicators, the establishment of a broad package task category and transaction mode matching analysis framework. Tian-Shu Song (2017) established a three-class object online assignment model in the environment of crowdsourcing. The model not only covers the tasks of crowdsourcing tasks, crowdsourcing workers and crowdsourcing work places, but also focuses on the dynamic environment. The stochastic threshold algorithm is established, and the optimal task allocation method is given. De-An Yu (2017) for the DTA algorithm allocation strategy single problem, the establishment of improved DTA model, multi-assigned tasks to high-skill level workers, do not assign tasks to low-skilled workers. The results show that the improved DTA algorithm is superior to DTA algorithm and random assignment algorithm. As for the crowdsourcing task pricing model, Trompette (2008) studied the economic model of community package, community collaboration, communication mechanism and incentive mechanism. The study pointed out that the problem of allocation of funds and asset transfer in crowdsourcing tasks was the core problem of crowdsourcing platform. What's more, DiPalantmo and Vojnovic (2009) proposed the use of full auction theory to model the crowd package, founding that by increasing the amount of reward money to promote the efforts of the 
participants and Archak and Sundararajan (2009) used the matching theory and utility theory in microeconomics to model the crowd package. The study found that the differential arrangement of the remuneration of the remuneration through the auction model can improve the profit of the contractor and make it more efficient crowds of resources in the process. Singer (2011) proposed a way to develop an optimized price in the case of limited budgets. Faridani (2011) proposed a contingency payment rule based on the Survival Analysis Model, which states that the contractor can be modeled by the non-homogeneous Poisson process model. This model can be used to predict the task completion time and generate pricing rules.

A complete set of crowdsourcing platforms must include assignments and pricing of tasks, and the above studies focus more on one part. Therefore, based on the principle of UTM projection, urban residents travel speed weighting method, k-means clustering, ant colony algorithm and comprehensive distance value theory, the assignment and pricing model of task is established, and reasonable packing strategy and pricing scheme are given.

\section{Task Allocation and Pricing Model}

The main factor influencing the task allocation strategy is the packing strategy of the task point, and the main factor influencing the pricing scheme is the expectation value of the length of the task and the regional economic level. After consulting a large number of related literatures, this article decided to choose K-means as a packaging strategy.

\subsection{Universal Transverse Mercator}

The UTM coordinate system is a planar Cartesian coordinate that converts latitude and longitude coordinates into UTM coordinates. This paper chooses the UTM to deal with the task points and the latitude and longitude coordinates of the members, which not only can find the plane distance between the two points more accurately, but also improve the calculation speed of K-means algorithm and ant colony algorithm.

Universal Transverse Mercator Formula (Li, 2013):

$$
\left\{\begin{aligned}
x= & 0.9996 s+\frac{1}{2} \cdot 0.9996 N \sin B \cos B l^{2}+\frac{1}{24} \cdot 0.9996 N t\left(5-t^{2}+9 \eta^{2}+4 \eta^{4}\right) \cos ^{4} B l^{4} \\
& +\frac{1}{720} \cdot 0.9996 N t\left(61-58 t^{2}+t^{4}+270 \eta^{2}-330 \eta^{2} t^{2}\right) \cos ^{6} B l^{6}+\cdots \\
y= & 0.9996 r l+\frac{1}{6} \cdot 0.9996 N\left(1-t^{2}+\eta^{2}\right) \cos ^{3} B l^{3} \\
& +\frac{1}{120} \cdot 0.9996 N\left(5-18 t^{2}+t^{4}+14 \eta^{2}-58 \eta^{2} t^{2}\right) \cos ^{5} B l^{5}+\cdots
\end{aligned}\right.
$$

\subsection{K-Means Clustering}

For a given data set containing $\mathrm{n} d$-dimensional data points $X=\left\{x_{1}, x_{2}, \cdots, x_{i}, \cdots, x_{n}\right\}$, where $x_{i} \in R^{d}$.The number of data subsets is $\mathrm{k}$. The $\mathrm{k}$-means clustering algorithm organizes the data objects into $\mathrm{k}$ divisions $C=\left\{c_{k}, i=1,2, \cdots, K\right\}$. Each division represents a class $C_{k}$, each class $C_{k}$ has a category center $\mu_{i}$. The distance between the interior point and the center of the cluster is calculated as the criterion of similarity and distance.

$$
J\left(c_{k}\right)=\sum_{x_{i} \in c_{k}}\left\|x_{i}-\mu_{k}\right\|^{2}
$$

The clustering goal is to minimize the sum of the squares of the total distances $J\left(c_{k}\right)=\sum_{k=1}^{K} J\left(c_{k}\right)$

$$
J\left(c_{k}\right)=\sum_{k=1}^{K} J\left(c_{k}\right)=\sum_{k=1}^{K} \sum_{x_{i} \in C_{k}}\left\|x_{i}-\mu_{k}\right\|^{2}=\sum_{k=1}^{K} \sum_{i=1}^{n} d_{k i}\left\|x_{i}-\mu_{k}\right\|^{2}
$$




$$
d_{k i}=\left\{\begin{array}{l}
1, x_{i} \in c_{i} \\
0, x_{i} \notin c_{i}
\end{array},\right.
$$

Obviously, according to the least squares method and the Lagrange theorem, the clustering center $\mu_{i}$ should be taken as the average of the class $c_{k}$ data points. The k-means clustering algorithm begins with an initial class a partition, and then assigns each data point to each category to reduce the sum of squares of the total distance. Since the sum of the squares of the total distance in the k-means clustering algorithm decreases with the increase in the number of categories (When $a=0, b=1$ ). Thus, the sum of squares of the total distance can only be achieved at a certain number of $\mathrm{k}$, the minimum value is obtained.

\subsection{The Ant Colony Algorithm}

The ant colony algorithm is a bionic algorithm used to find the probability of the optimal path, compared with the genetic algorithm, simulated annealing algorithm, ant colony algorithm is not easy to fall into local optimum but easy to find the global optimal solution. Because each point package contains many different tasks, this paper using the ant colony algorithm to solve the shortest Hamiltonian circuit. The specific implementation steps for solving the Hamiltonian algorithm are as follows.

Step 1 Parameter initialization. Set time $t=0$ and cycle times $N_{c}=0$, Set the maximum cycle time $N_{c_{\max }}$, substitute the number of ants $m$ to task point $n$, making the amount of information initialized on each edge of the graph, $\tau_{i j}(t)=$ const, where $c o n s t$ is a constant, and at the initial moment, $\Delta \tau_{i j}(0)=0$.

Step 2 Calculate the cycle times $N_{c} \leftarrow N_{c}+1$.

Step 3 Set the ant taboo index $k=1$.

Step 4 Calculate the number of ants $k \leftarrow k+1$.

Step 5 The ant individual selects and heads to the element (task) $j$ according to the probability of the state transition probability formula, where $j \in\left\{C-t a b u_{k}\right\}$.

$$
\begin{aligned}
& \eta_{i j}(t)=\frac{1}{d_{i j}} \\
& \tau_{i j}(t+n)=(1-\rho) \cdot \tau_{i j}(t)+\Delta \tau_{i j}(t) \\
& \Delta \tau_{i j}(t)=\sum_{k=1}^{m} \tau_{i j}^{k}(t) \\
& \rho_{i j}^{k}(t)=\left\{\begin{array}{cc}
\frac{\left[\tau_{i j}(t)\right]^{\alpha} \cdot\left[\eta_{i k}(t)\right]^{\beta}}{\sum_{s \subset \text { allowed }}\left[\tau_{i j}(t)\right]^{\alpha} \cdot\left[\eta_{i k}(t)\right]^{\beta}}, & \text { if } j \in \text { allowed }_{k} \\
0, & \text { otherwise }
\end{array}\right.
\end{aligned}
$$

where, $k$ is the number of the ants;

$d_{i j}$ is the distance between two adjacent tasks;

$C=\left\{c_{1}, c_{2}, \cdots, c_{n}\right\}$ is the collection of tasks $n$; 
$\eta_{i j}(t)$ is heuristic function;

$\tau_{i j}$ is the amount of pheromone deposited for transition from task $\mathrm{i}$ to task $\mathrm{j}$;

$\rho_{i j}^{k}(t)$ is the transfer rate of the ant $k$, which transfers from the task i to task j;

$t a b u_{k}$ is the taboo table, recording the tasks that ant $k$ have passed at time t;

allowed $_{k}=C-$ tabu $_{k}$ is the next step allowed the selected task by the ant;

$\alpha$ is heuristic factor;

$\beta$ is expected heuristic factor.

Step 6 Adjust the taboo pointer, in other words, add the tasks the ant have passed to the taboo table.

Step 7 Update the amount of information according to Equation(1) and Equation(2).

Step 8 If the end condition is satisfied, that's to say, the cycle times $N_{c} \geq N_{c \max }$, end the loop and output the calculation result. Otherwise, clear the taboo table and turn to Step 2.

\subsection{Psychological Surplus Value Model}

In real life, members have a different standard of evaluation criteria, this standard directly determines whether to complete the task. Taking into account the completion of the process of the existence of the members of the time cost, and the distribution of the task in the market will lead to members of the project to affect the completion of the task, so this definition

$$
S=P-F \cdot T
$$

where, $S$ is the psychological residual value of the member, $T$ is the cost of personal travel time, $F$ is a non-shopping factor. When $S \geq 0$, members of the psychological surplus is positive, members have the motivation to complete the task, the task is completed; when $S<0$, members of the psychological surplus is negative, members lack the power to complete the task, resulting in no one task.

For personal travel time cost $\mathrm{T}$, this paper analyzes the working day, regional GDP, traffic congestion, the difficulty of the actual completion of the task. Definition

$$
T=\mathrm{a} \times b \times\left(\frac{L_{i}}{\sum_{i=1}^{4} \mu_{i} V_{i}}+\mathrm{h}\right) \times V_{o t}
$$

In the formula, a is the traffic jam factor, $b$ is the actual path length factor, $L_{i}$ is a long road trip, which is the minimum Hamiltonian circuit, $\mu_{i}$ is the $i$ way to travel in the residents travel structure proportion, $V_{i}$ is the average speed of the $i$ mode, $\mathrm{h}$ is the task execution time, $V_{o t}$ is single a member of the value of time.

\section{Numerical Examples}

\subsection{UTM Processing Results}

Introduce the UTM coordinate system to deal with the latitude and longitude coordinates of the task point, get the following UTM coordinates 
Table 1. Clustering center UTM coordinates and City geolocation data

\begin{tabular}{cccc}
\hline Clustering center label & UTM x-coordinate & UTM y- coordinate & City \\
\hline 1 & 734535.934 & 2573011.821 & Shenzhen \\
2 & 772868.880 & 2552363.926 & Shenzhen \\
3 & 810362.269 & 2509395.302 & Shenzhen \\
$\ldots \ldots$ & $\ldots \ldots . .$. & Dongguan \\
625 & 823421.4975 & $\ldots \ldots$ & Dongguan \\
\hline
\end{tabular}

\subsection{K-means Processing Results}

First determine the number of clusters, the establishment of the number of clusters on the mathematical formula:

$$
N_{\text {cluster }}=N_{\text {total }}\left[1-\left(\frac{1}{2}\right)^{n}\right] \quad(n=1,2, \cdots, n)
$$

In the formula, $N_{\text {cluster }}$ is the number of cluster centers, $N_{\text {total }}$ is the total number of samples.

By MATLAB test, it is concluded that when $b=0$, the clustering effect is the best, so the final cluster center number is $N_{\text {cluster }}=835 \times\left[1-\left(\frac{1}{2}\right)^{2}\right] \approx 626$

The task points are packed by K-means clustering algorithm. The clustering effect is shown in Figure 1.

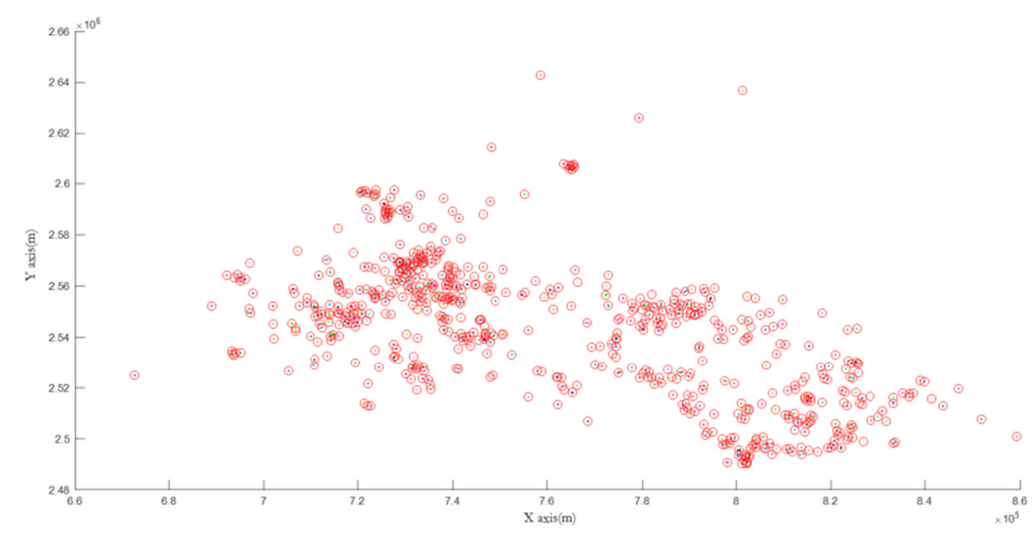

Figure 1. Task Point Clustering Center Map

The correspondence between the data sample and the clustering center is shown in Table 2 . 
Table 2. The Corresponding Task Number Corresponds to the Cluster Center

\begin{tabular}{cccc}
\hline Task Number & Latitude & Longitude & Clustering Number \\
\hline A0001 & 22.56614225 & 113.9808368 & 319 \\
A0002 & 22.68620526 & 113.9405252 & 164 \\
A0003 & 22.57651183 & 113.957198 & 271 \\
$\ldots \ldots$ & $\ldots \ldots$ & $\ldots \ldots$ & $\ldots \ldots$ \\
A0834 & 23.06367398 & 113.7711884 & 57 \\
A0835 & 23.12329431 & 113.1103823 & 483 \\
\hline
\end{tabular}

\subsection{Ant Colony Algorithm Processing Results}

Find the Hamiltonian circuit which belongs to the same cluster center task point. Taking a cluster center as an example, the Hamiltonian circuit with its assigned task is shown in Figure 2. The ant colony algorithm is used to solve the shortest path $L_{\text {min }}$, and its optimization, the results shown in Figure 3.

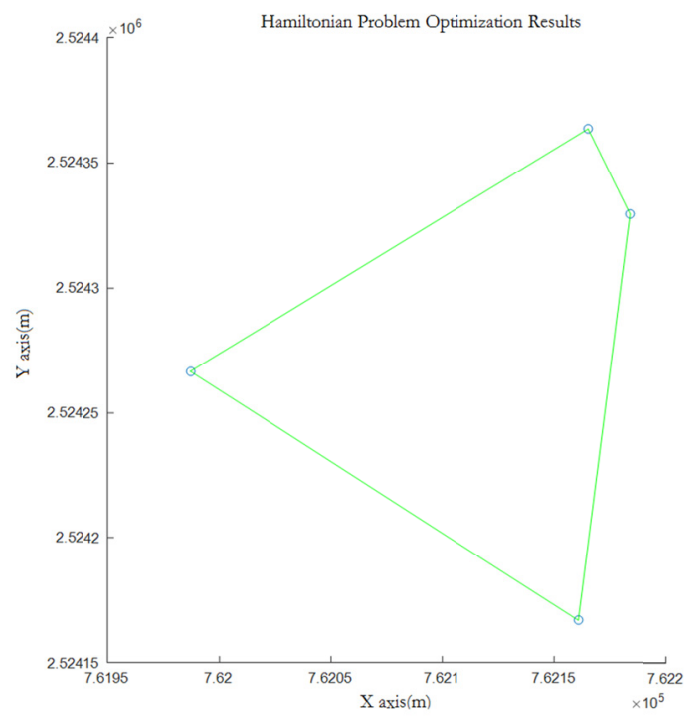

Figure 2. Hamiltonian Circuit of Task Points

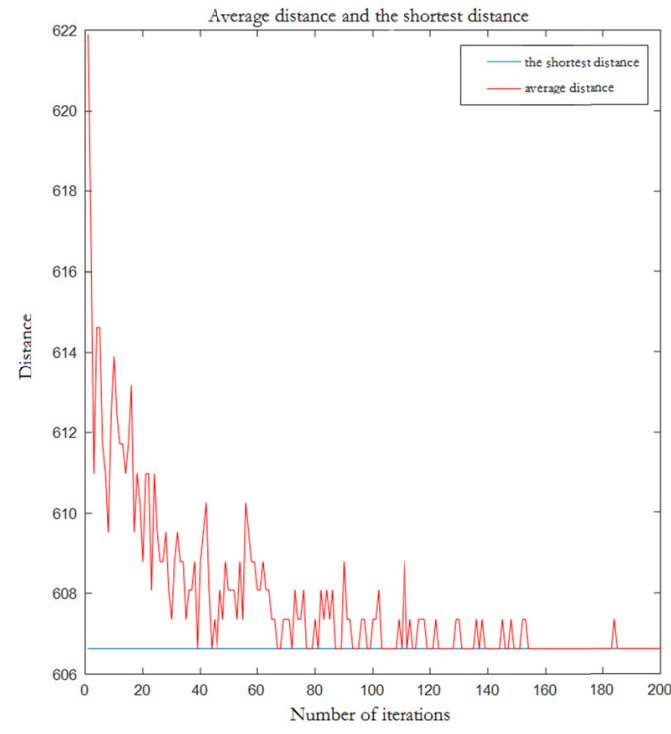

Figure 3. Ant Colony Algorithm Optimization

In the actual situation, when the distance from the task points are more than $5 \mathrm{~km}$, the members will be lack of motivation to complete the task, therefore, based on the shortest Hamiltonian road integrated distance $L_{\min }+5 \mathrm{~km}$, to get the cluster center corresponding to the shortest Hamiltonian integrated clustering data as shown in Table 3. 
Table 3. Combined with the final distance of the Hamiltonian circuit

\begin{tabular}{cc}
\hline Clustering number & Integrated distance \\
\hline 1 & 5.00000000 \\
2 & 6.00012131 \\
3 & 5.00000000 \\
$\ldots \ldots$ & $\ldots \ldots$ \\
625 & 5.00000000 \\
626 & 5.00000000 \\
\hline
\end{tabular}

\subsection{Psychological Surplus Value Model Processing Results}

\subsubsection{The Determination of the Remaining Factors of the Model}

$$
\left\{\begin{array}{l}
S=P-F \cdot T \\
T=\mathrm{a} \times b \times\left(\frac{L_{i}}{\sum_{i=1}^{4} \mu_{i} V_{i}}+\mathrm{h}\right) \times V_{o t}
\end{array}\right.
$$

1) Factor F: within the allowable range of the error, the proportion of the shopping factor in the Pearl River Delta area is about $10 \%$, so the non-shopping factor $\mathrm{F}$ is $90 \%$. (Jiang 2014)

2) Factors $a, b$ and $h$ : taking into account the actual route there is a clogging situation, and the two tasks are not between the straight line. After checking the relevant literature to determine the traffic jam factor a is 1.2 , the actual road length factor $b$ is 1.5 , and the implementation of the task takes some time, we found $h$ for $1 / 3$ hours.

3) Factors $\mu_{i}$ and $V$ : short distance residents travel mode structure shown in Table 4, different travel speed as shown in Table 5. (Chang 2007).

Table 4. Short Distance Residents Travel Mode Structure

\begin{tabular}{cccccc}
\hline & Walk & Bicycles & Car or Taxi & Bus (Subway) & others \\
\hline Proportion & $46 \%$ & $32 \%$ & $8 \%$ & $9 \%$ & $5 \%$ \\
\hline
\end{tabular}

Table 5.The Speed of different vehicles

\begin{tabular}{ccccc}
\hline & Walk & Bicycles & Car or Taxi & Bus (Subway) \\
Speed $(\mathrm{km} / \mathrm{h})$ & 4 & 15 & 60 & 40 \\
\hline
\end{tabular}

4) Factor $V_{o t}$ : in view of the member unit time value and the per capita GDP and personal work time, $V_{o t}=\frac{\overline{G D P}}{D \times H}$ is defined. In the formula, $\overline{G D P}$ is GDP per person, $\mathrm{D}$ is the annual working day, and $\mathrm{H}$ is the working hours per day. The value of $\overline{G D P}$ is shown in Table 6. For D, excluding the annual double Sunday and statutory holidays, you can get $365-11=250$, that is, D for 250 days. As for $\mathrm{H}$, according to the actual situation can be defined as 8 hours. 
Table 6. Economic situation of major cities in Guangdong Province, China

\begin{tabular}{cccc}
\hline Prefecture-level city & 2016 GDP (billion yuan) & 2016 per capita GDP (yuan) & $\begin{array}{c}\text { Per capita monthly income } \\
\text { (yuan) }\end{array}$ \\
\hline Guangzhou & 19611 & 145254 & 6060 \\
Shenzhen & 19492 & 171305 & 6940 \\
Foshan & 8630 & 116141 & 4890 \\
Dongguan & 6275 & 82719 & 5200 \\
\hline
\end{tabular}

\subsubsection{Model Solving}

The psychological value surplus model is solved to get the packing task pricing data as shown in table 7 .

Table 7. Final Task Pricing

\begin{tabular}{cccc}
\hline Clustering number & Task pricing (yuan) & Clustering number & Task pricing (yuan) \\
\hline 1 & 103.2310051 & 623 & 49.84773072 \\
2 & 112.4579919 & 624 & 49.84773072 \\
3 & 103.2310051 & 625 & 49.84773072 \\
$\ldots \ldots$ & $\ldots \ldots$ & 626 & 49.84773072 \\
\hline
\end{tabular}

As it turns out, in fact, the total amount of the project has been completed the total price of 57,707.5 yuan, while through the psychological surplus value model to develop the program's total price of 58,607.6 yuan, and in ensuring the completion of all tasks under the conditions, the total price of packaging only 54395.6 yuan, only increases by $1.56 \%$ of the cost, however, the task completion increases by $37.485 \%$. Indicating that through the psychological surplus value model for each task targeted pricing can help to improve capital utilization.

\section{Conclusion}

This article is rigorous, from the task allocation and task pricing point of view cut into the establishment of the model. In the task allocation, from the distribution strategy of the distribution of the reality of the situation, the use of k-means clustering algorithm to package the task point. Taking into account the elliptical latitude and longitude coordinates on the distance between the two points is not the European distance, the introduction of UTM coordinate system on the task point latitude and longitude coordinates for processing. At the same time, the establishment of the clustering center quantity formula, on the basis of the effective packing task point, not only reduces the error of the $\mathrm{k}$ value determined by the original empirical judgment method, but also improves the running speed of the algorithm. In the task pricing, combined with the economic theory, starting from the psychological will of members, to build a psychological surplus value model. The ant colony algorithm is used to calculate the factor value of the shortest Hamiltonian circuit, and then the other factor values of the model are determined according to the actual situation, so as to solve the problem of pricing and completion when the task is completed. The results show that the total price after packaging is only 54395.6 yuan, which is only $1.56 \%$ higher than the original pricing, and the task completion is increased by $37.485 \%$, which shows that through the psychological surplus value model for each task To carry out targeted pricing, while improving the capital utilization rate can greatly improve the task completion rate, but also shows the validity and rationality of the model.

\section{References}

DiPalantino, D., Vojnovic, M. (n.d.). Crowdsourcing and all-pay auctions. In Proceedings of the l0th ACM conference on Electronic commerce. http://dx.doi.org/10.1145/1566374.1566392

Faradani, S., Hartmann, B., \& Ipeirotis, P. G. (2011). What's the Right Price? Pricing Tasks for Finishing on Time. Human Computation. from 
https://www.aaai.org/ocs/index.php/WS/AAAIW11/paper/viewFile/3994/4269

Singer, Y., \& Mittal, M. (2011). Pricing Tasks in Online Labor Markets. In Proc. Of Human Computation. Retrieved from https://www.aaai.org/ocs/index.php/WS/AAAIW11/paper/viewFile/4001/4266/

Archak, N., Sundararajan, A. (2009). Optimal Design of Crowdsourcing Contest. ICIS. Retrieved from http://pages.stern.nyu.edu/ narchak/optimalCrowdsourcingDesign.pdf

Trompette, P., Chanal, V., Pelissier, C. (2008). Crowdsourcing as a way to access external knowledge for innovation. In 24th EGOS Colloquium. Retrieved from https://halshs.archives-ouvertes.fr/halshs-00367373

Mengzhi, X., \& Qianming, Z. (2017). Study on Quantitative Research on Opportunity Cost. Knowledge Economy, (13), 17-19. http://dx.chinadoi.cn/10.3969/j.issn.1007-3825.2017.13.009

Bing, W., \& Qihui, S. (2003). Consumer surplus theory research summary. Economic Aspect, (12), 59-62. http://dx.chinadoi.cn/10.3969/j.issn.1007-7685.2003.12.016

Suhui, W., Ying, C., Yanning, Z., \& Yuntao, P. (2011). K-means algorithm research. Modern Library and Information Technology, (5), 28-35. http://dx.chinadoi.cn/10.11925/infotech.1003-3513.2011.05.05

Zhifei, S. (2013). TSP problem based on ant colony algorithm. Jiangxi University of Science and Technology. Retrieved from http://xuewen.cnki.net/CJFD-HERZ201702028.html

Siyi, S. (2016). China's consumption level of the impact of empirical research. Finance and Economics, (06), 39-41. Retrieved from http://www.cqvip.com/qk/71030x/2016003/668233966.html

Chaofan, C. (2007). Urban residents short-distance travel behavior research. Beijing Jiaotong University. Retrieved from http://cdmd.cnki.com.cn/Article/CDMD-10004-2008049213.htm

Haiyan, J., Xuemei, Z., Zebin, S., \& Lingling, W. (2014). Chinese residents of the traffic trend of differentiation and the implications of traffic fairness. Planner, 30(1), 94-100. http://dx.chinadoi.cn/10.3969/j.issn.1006-0022.2014.01.018

Guoyi, L., \& Chuguang, Y. (2013). UTM Projection and Its Deformation Analysis. Geospatial Information, 11 (06), 80-83 + 10. http://dx.chinadoi.cn/10.11709/j.issn.1672-4623.2013.06.028

Qian, W., Cheng, W., Zhenyuan, F. (2012). Study on K-means clustering algorithm. Electronic Design \& Engineering, 20(7), 21-24. http://dx.chinadoi.cn/10.3969/j.issn.1674-6236.2012.07.008

Ling, Q. (2004). Improvement and Application of Ant Colony Algorithm. Yangzhou University,

Tianshu, S., Yongxin, T., Libin, \& Ke, X. (2017). Online task assignment for 3 classes of objects in space crowdsourcing environment. Software Journal, 28(3), 611-630. Retrieved from http://www.jos.org.cn/html/2017/3/5166.htm

Jun, W., Haozhe, C., \& Jiaqi, Z. (2015). Study on Task Matching and Transaction Matching of Rising Package Platforms. Science and Technology Progress and Countermeasures, 32(14), 6-11. http://dx.chinadoi.cn/10.6049/kjjbydc.2015030643

Dean, Y. (2017). The improvement and application of crowdsman task allocation algorithm. Dalian Maritime University. Retrieved from http://cdmd.cnki.com.cn/Article/CDMD-10151-1017054443.htm

\section{Copyrights}

Copyright for this article is retained by the author(s), with first publication rights granted to the journal.

This is an open-access article distributed under the terms and conditions of the Creative Commons Attribution license (http://creativecommons.org/licenses/by/4.0/). 